\title{
Improved standardisation of training needed to achieve the potential of handheld echocardiography
}

\author{
Bruno Ramos Nascimento [1] , ${ }^{1,2}$ Andrea Zawacki Beaton (i) 3,4
}

There remains no doubt that handheld echocardiography has a place among tools used to improve the diagnosis of structural heart disease. The last decade has seen advances in image quality, battery life, portability, file formats and available features, all with increasingly lower costs. ${ }^{1}$ Handheld ultrasound devices (HUD) have now been used in a wide variety of settings, from emergency departments in the USA to primary healthcare clinics in remote parts of sub-Saharan Africa. Users have been just diverse, ranging from highly skilled echocardiographers to those with only days of ultrasound training. With this diversity of scope and skill, evaluation of the accuracy of these devices is both complex and critical, requiring a robust compilation of available evidence.

The current systematic review and meta-analysis aimed to evaluate the diagnostic accuracy of HUD to detect left ventricular systolic dysfunction and to stratify accuracy by the experience of the user. Over 6000 participants from 33 included studies demonstrated that experienced operators could accurately predict reduced left ventricular ejection fraction (LVEF), wall motion abnormalities, LV dilatation and LV hypertrophy, with pooled sensitivities ranging from $85 \%$ to $89 \%$, and pooled specificities from $91 \%$ to $98 \%$, as compared with conventional transthoracic echocardiography.

High performance of experts using handheld ultrasound is less a test of the user (already known to be highly skilled) and more a test of technology, ultimately boosted by the incorporation of high-end features. While each handheld ultrasound

${ }^{1}$ Serviço de Cardiologia e Cirurgia Cardiovascular, Hospital das Clinicas da Universidade Federal de Minas Gerais, Belo Horizonte, MG, Brazil

${ }^{2}$ Centro de Telessaúde, Hospital das Clínicas da Universidade Federal de Minas Gerais, Belo Horizonte, MG, Brazil

${ }^{3}$ The Heart Institute, Cincinnati Childrens Hospital Medical Center, Cincinnati, OH, USA

${ }^{4}$ School of Medicine, University of Cincinnati, Cincinnati, $\mathrm{OH}$, USA

Correspondence to Dr Bruno Ramos Nascimento, Serviço de Cardiologia e Cirurgia Cardiovascular, Hospital das Clinicas da Universidade Federal de Minas Gerais, Belo Horizonte, MG, Brazil;

ramosnas@gmail.com has its strengths and weaknesses, and new devices are rapidly entering the market, it is safe to say that in general, these results confirm that HUD have advanced to the point, where they can be used to reliably evaluate LV function in different scenarios. This assertion is backed by a 2011 American Society of Echocardiography consensus statement that stated LV dilation, hypertrophy and systolic function, left and right atrial enlargement, pericardial effusion and vena cava size could all be accurately evaluated by handheld ultrasound in clinical practice. ${ }^{1}$

The more interesting question posed in this systematic review addresses the variability of performance among users, which reflects more on training, than it does on technology. In this meta-analysis, Jenkins and colleagues ${ }^{2}$ concluded that while nonexperienced operators were able to detect cardiac LV abnormalities with reasonable sensitivity $(68 \%-83 \%)$ and specificity (87\%-95\%), there was a significant difference in diagnostic accuracy between those with and those without a high-level experience. This finding is not surprising, but of high importance for both research and practice.

Addressing training and maintenance of competency in non-expert or nontraditional users is critical. One of the greatest potentials for handheld ultrasound, including echocardiography, is to extend diagnostic capacity to areas where standard ultrasound or echocardiography_for their multiple purposes-and expert users are not available. In fact, the World Health Organization estimates that access to ultrasound could meet $90 \%$ of imaging needs in the primary care setting in low-income and middle-income countries (LMICs). ${ }^{3}$ And there is no doubt that ultrasound research and clinical use is undergoing exponential growth in LMICs. A recent systematic review of trends in ultrasound use in LMICs included over 4000 ultrasound studies between 2010 and 2018 with the majority being from southeast Asia and sub-Saharan Africa. ${ }^{4}$

The challenge then is to develop reliable methods for task-shifting that incorporate rigorous, yet efficient training protocols that enable scale-up of these technologies.
Many studies have looked at training non-expert users in handheld echocardiography with variable results. One such study included brief training of 12 nonexperts in Kenya used the Focus Assessed Transthoracic Echocardiography protocol which included two on-line learning modules, a 1-day hands-on workshop and a 3-month follow-on period where trainees were to perform 10 directly supervised and 10 additional scans. In this case, over two-thirds of trainee images were judged to be poor quality at the end of the training period assessment and the team concluded that additional supervised practice limited gains in skills. ${ }^{5}$

In contrast, a study in Northern Uganda included eight novice trainees in an innovative curriculum that included remote mentorship to build competency for heart failure diagnosis and broad categorisation by echocardiography. In this study, participants completed an online course (Wired International), a 2-day hands on workshop, and a 2-month period of remote mentorship, where each trainee was required to submit and receive feedback on at least 30 imaging examinations. While only 4 of 8 trainees completed all of the phases and passed competency testing, again reflecting the challenge of finding time for skills acquisition, those who did complete the programme had 93\% concordance with expert interpretation over a 4-month independent scanning period. ${ }^{6}$ Although with a different scope-diagnosis of latent rheumatic heart valve disease-a similarly successful training approach was reported in Brazil: six non-experts with different backgrounds (from nurses to medical students) completed a 3-week remote and hand-on curriculum on HUD image acquisition with and interpretation, and their performance was tested in a schoolscreening environment compared with a reference standard echo performed by cardiologists. Overall sensitivity of the simplified approach was $83 \%$ and overall specificity $85 \%$. $^{7}$ This was part of a taskshifting strategy currently in expansion to other scenarios, such as primary care and secondary cardiovascular care.

These very different outcomes highlight the importance of the both the training curriculum and the training environment. To effectively scale up handheld echocardiography, there will need to be an investment in standardised, replicable training programmes and competency assessment. There is growing global enthusiasm for this approach with organisations emerging such as the Point-of-Care Ultrasound Certification Academy and the Global Ultrasound Institute, though work remains 
around scale, access, quality assurance and accreditation of these and other bodies.

Innovation in artificial intelligence may also improve both the training of nonexperts to conduct echocardiograms and to interpret them. A new FDA-approved navigational guidance software used deeplearning and neural networks to guide users into the correct probe position for standard echocardiography views, also applicable to HUD. Study of this technology showed non-expert users able to achieve nearly 99\% acquisition of diagnostic quality images with only 1 hour of didactic training and nine supervised practice scans. ${ }^{8}$ Deep-learning algorithms have also shown encouraging results for the measurement of ventricular function and structure, and an automated software for calculating LVEF has been approved by the FDA and tested in an integrated format on handheld echocardiography machines. ${ }^{8}$ More recently, novel deeplearning algorithms are being tested also for more specific diagnoses, such as subtle valve findings associated with subclinical rheumatic heart disease. ${ }^{9}$ There is no doubt these technologies will continue to improve and expand and could play a pivotal role in global scale-up of handheld echocardiography.

In summary, the use of HUD for different purposes will continue to expand in both high-resource and low-resource settings, with growing technological implementation. This is incredibly good news for those who live in areas where diagnostics remain sparse and diagnosis often comes too late. However, it also compels careful consideration and action to develop standards and resources for training and competency testing that are both practical and feasible in lower resourced settings and ensure that the provision of diagnostic equipment has the highest impact in the places that need it the most. More than ever, robust accuracy data as presented in this metaanalysis, aligned with practicability of the method and the developing training/ educational resources, are placing HUD as a fifth pillar of modern bedside examination: inspection, palpation, percussion, auscultation and, now, insonation.

Twitter Bruno Ramos Nascimento @ramosnas

Funding $\mathrm{Dr} \mathrm{BN}$ is supported in part by $\mathrm{CNPq}$ (Bolsa de produtividade em pesquisa, 312382/2019-7), by the Edwards Lifesciences Foundation (Improving the Prevention and Detection of Heart Valve Disease Across the Lifespan, 2021) and by FAPEMIG (grant APQ-0 00 627-20).

Competing interests None declared.

Patient and public involvement Patients and/or the public were not involved in the design, or conduct, or reporting, or dissemination plans of this research.

Patient consent for publication Not applicable.

Provenance and peer review Commissioned; externally peer reviewed.

\section{0 \\ OPEN ACCESS}

Open access This is an open access article distributed in accordance with the Creative Commons Attribution Non Commercial (CC BY-NC 4.0) license, which permits others to distribute, remix, adapt, build upon this work non-commercially, and license their derivative works on different terms, provided the original work is properly cited, appropriate credit is given, any changes made indicated, and the use is non-commercial. See: http:// creativecommons.org/licenses/by-nc/4.0/.

(c) Author(s) (or their employer(s)) 2021. Re-use permitted under CC BY-NC. No commercial re-use. See rights and permissions. Published by BMJ.

\section{Check for updates}

To cite Ramos Nascimento B, Zawacki Beaton A. Heart 2021;107:1772-1773.
Published Online First 27 September 2021

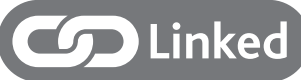

- http://dx.doi.org/10.1136/heartjnl-2021-319561

Heart 2021;107:1772-1773.

doi:10.1136/heartjnl-2021-319945

\section{ORCID iDs}

Bruno Ramos Nascimento http://orcid.org/0000-00025586-774X

Andrea Zawacki Beaton http://orcid.org/0000-00024963-355X

\section{REFERENCES}

1 Chamsi-Pasha MA, Sengupta PP, Zoghbi WA. Handheld echocardiography: current state and future perspectives. Circulation 2017;136:2178-88.

2 Jenkins S, Alabed S, Swift A, et al. Diagnostic accuracy of handheld cardiac ultrasound device for assessment of left ventricular structure and function: systematic review and meta-analysis. Heart 2021:1826-34.

3 Maksimovic R, Berumen AV. Innovative technology in addressing global health issues: the who perspective. Geneva, Switzerland: World Health Organization (WHO/ HQ/HSS/EHT/DIM), 2011.

4 Stewart KA, Navarro SM, Kambala S, et al. Trends in ultrasound use in low and middle income countries: a systematic review. Int J MCH AIDS 2020;9:103-20.

5 Waweru-Siika W, Barasa A, Wachira B, et al. Building focused cardiac ultrasound capacity in a lower middleincome country: a single centre study to assess training impact. Afr J Emerg Med 2020;10:136-43.

6 DeWyer A, Scheel A, Otim IO, et al. Improving the accuracy of heart failure diagnosis in low-resource settings through task sharing and decentralization. Glob Health Action 2019;12:1684070.

7 Beaton A, Nascimento BR, Diamantino AC, et al. Efficacy of a standardized computer-based training curriculum to teach echocardiographic identification of rheumatic heart disease to Nonexpert users. Am J Cardiol 2016;117:1783-9.

8 Narang A, Bae R, Hong $\mathrm{H}$, et al. Utility of a DeepLearning algorithm to guide novices to acquire Echocardiograms for limited diagnostic use. JAMA Cardiol 2021;6:624-32.

9 Martins JFBS, Nascimento ER, Nascimento BR, et al. Towards automatic diagnosis of rheumatic heart disease on echocardiographic exams through video-based deep learning. J Am Med Inform Assoc 2021:28:1834-42. 